\title{
Guidelines
}

\section{Endoscopic Ultrasound-Guided Ablation of Focal Pancreatic Lesions: The GRUPUGE Perspective}

\author{
Miguel Bispo $^{a} \quad$ Ana Caldeira $^{b}$ Sílvia Leite ${ }^{c}$ Susana Marques ${ }^{a}$ \\ Teresa Moreira $^{d}$ Pedro Moutinho-Ribeiro ${ }^{e}$ Nuno Nunes $^{f}$ \\ Portuguese Group for Ultrasound in Gastroenterology (GRUPUGE) \\ a Department of Gastroenterology and Digestive Endoscopy, Champalimaud Foundation, Lisbon, Portugal; \\ ${ }^{\mathrm{b}}$ Department of Gastroenterology, Hospital Amato Lusitano, Castelo Branco, Portugal; ' $D e p a r t m e n t ~ o f$ \\ Gastroenterology, Hospital da Senhora da Oliveira, Guimarães, Portugal; ${ }^{d}$ Department of Gastroenterology, Centro \\ Hospitalar Universitário do Porto - Hospital de Santo António, Porto, Portugal; ' Department of Gastroenterology, \\ Centro Hospitalar e Universitário São João, Porto, Portugal; ${ }^{f}$ Department of Gastroenterology, Hospital do Divino \\ Espírito Santo, Ponta Delgada, São Miguel, Portugal
}

\section{Keywords}

Chemoablation · Endoscopic ultrasound-guided ablation · Pancreatic mucinous neoplasm · Pancreatic neuroendocrine tumour $\cdot$ Pancreatic cyst $\cdot$ Radiofrequency ablation

\section{Abstract}

Focal pancreatic lesions include a heterogeneous group of solid and cystic lesions, with different natures and variable clinical, imagiological, and pathological characteristics. Several endoscopic ultrasound (EUS)-guided ablative techniques have been tested during the last decade for the treatment of these pancreatic lesions, mostly consisting of the injection of ablative agents and, more recently, radiofrequency ablation. The most encouraging EUS-guided ablation outcomes are being reached in the treatment of some pancreatic cystic neoplasms and small $(\leq 2 \mathrm{~cm})$ pancreatic neuroendocrine tumours ( $p N E T s$ ). Data supporting a potential role of ablative therapies in the treatment of pancreatic ductal adenocarcinoma is still lacking. In this article, GRUPUGE presents an updated perspective of the potential role of EUS-guided ablation for the treatment of pancreatic cystic neoplasms and pNETs, addressing the selection criteria and technical issues of different techniques and analysing recent data on their safety and efficacy.

(c) 2020 Sociedade Portuguesa de Gastrenterologia Published by S. Karger AG, Basel

\section{Ablação Guiada por Ecoendoscopia de Lesões Focais do Pâncreas: Perspectiva do GRUPUGE}

\section{Palavras Chave}

Quimio-ablação · Ablação guiada por ecoendoscopia · Neoplasia mucinosa pancreática · Tumor neuroendócrino pancreático · Quisto pancreático . Ablação por radiofrequência

\section{Resumo}

As lesões focais do pâncreas integram grupos heterogéneos de lesões sólidas e quísticas, de diferentes naturezas e com características clínicas, imagiológicas e patológicas variáveis. Na última década foram avaliadas diversas técnicas ablativas guiadas por ecoendoscopia para o trata-

\begin{tabular}{ll}
\hline KARGER & $\begin{array}{l}\text { (c) 2020 Sociedade Portuguesa de Gastrenterologia } \\
\text { Published by S. Karger AG, Basel }\end{array}$ \\
karger@karger.com & $\begin{array}{l}\text { This article is licensed under the Creative Commons Attribution- } \\
\text { NonCommercial-NoDerivatives 4.0 International License (CC BY- } \\
\text { NC-ND) (http://www.karger.com/Services/OpenAccessLicense). } \\
\text { www.karger.com/pjg } \\
\text { Usage and distribution for commercial purposes as well as any dis- } \\
\text { tribution of modified material requires written permission. }\end{array}$ \\
BOPEN ACCESS &
\end{tabular}


mento destas lesões pancreáticas focais, consistindo maioritariamente na injeção de agentes ablativos e, mais recentemente, na ablação por radiofrequência. Os resultados mais promissores das técnicas ablativas guiadas por ecoendoscopia têm surgido no tratamento de algumas lesões quísticas do pâncreas e pequenos tumores neuroendócrinos pancreáticos $(\leq 2 \mathrm{~cm})$. Ainda existem poucos dados a suportar um potencial papel das terapêuticas ablativas no tratamento do adenocarcinoma ductal do pancreas. No presente artigo o GRUPUGE apresentada uma perspectiva atual do potencial papel da ablação guiada por ecoendoscopia no tratamento de neoplasias quísticas do pâncreas e de tumores neuro-endócrinos pancreáticos, focando aspectos relativos à seleção dos doentes, questões técnicas dos vários procedimentos disponíveis e analisando dados recentes relativos à sua segurança e eficácia.

๑) 2020 Sociedade Portuguesa de Gastrenterologia Publicado por S. Karger AG, Basel

\section{Introduction}

Asymptomatic focal pancreatic lesions (cystic and solid) are being increasingly diagnosed because of the advances in conventional imaging (abdominal ultrasound, CT and MRI) [1,2]. The detected lesions include mostly small pancreatic cystic neoplasms (most commonly, branch-duct intraductal papillary mucinous neoplasms [BD-IPMNs]) and pancreatic neuroendocrine tumours (pNETs) that are usually well-differentiated $[2,3]$. Although these incidentally detected focal pancreatic lesions ("incidentalomas") may harbour some malignant potential, the majority will probably do no harm to patients [2-4]. The natural history of these incidentally detected pancreatic lesions is variable and, in most cases, difficult to predict when based on morphologic and endoscopic ultrasound fine-needle aspiration/fine-needle biopsy (EUS-FNA/FNB) findings, which makes treatment decisions challenging [4].

Presently, there are 2 main approaches towards these lesions in clinical practice: surveillance or surgery [4]. For focal pancreatic lesions containing malignancy or considered to have a high risk of malignant transformation, surgery is indicated [2-4]. However, opting for surgery based on concerns about malignancy has at times been excessive, and as we have come to understand the natural course of most of these lesions better, management has gradually become more conservative $[4,5]$.

In the management of incidentally discovered pancreatic cystic neoplasms and small pNETs, it would be desir-

EUS-Guided Ablation of Focal Pancreatic Lesions able to have an "intermediate option," somewhere between surveillance and surgery. A safe and effective minimally invasive treatment option, like EUS-guided ablation, would have a major impact in clinical practice, as we could:

1 Offer treatment to potentially pre-cancerous lesions (e.g., mucinous cysts and small pNETs) and some symptomatic lesions (e.g., functional pNETs).

2 Avoid surgery-associated morbidities.

3 Prevent progression to malignancy.

The most encouraging EUS-guided ablation outcomes are being reached in the treatment of some pancreatic cystic neoplasms and small $(\leq 2 \mathrm{~cm})$ pNETs [6-8]. Data supporting a potential role of ablative therapies in the treatment of pancreatic ductal adenocarcinoma is still lacking [9].

In this article, the Portuguese Group for Ultrasound in Gastroenterology (GRUPUGE) presents a perspective of the potential role of EUS-guided ablation in the treatment of pancreatic cystic neoplasms and pNETs, addressing the selection criteria and technical issues of the different techniques and analysing emerging data on their efficacy and safety. A systematic literature search was performed until January 2020, using PubMed, MEDLINE, Scopus, and Google, using the key words "chemo-ablation," "endoscopic ultrasound-guided ablation," "pancreatic mucinous neoplasm," "pancreatic neuroendocrine tumour," "pancreatic cyst" and "pancreatic radiofrequency ablation." Prospective/comparative studies and international consensus statements/management guidelines were preferred.

\section{Ablation of Pancreatic Cystic Neoplasms}

The aim of the treatment of pancreatic cystic neoplasms is to prevent malignant progression. The risk of malignancy that a mucinous cyst yields is linked to the presence of high-risk or worrisome features [10]. Current guidelines indicate that surgery should be considered in the presence of "high-risk stigmata," even in asymptomatic cysts, i.e., a contrast-enhanced mural nodule (Fig. 1) or a major dilatation of the main pancreatic duct (MPD, $\geq 10$ mm; Fig. 2) [10].

Patients with "worrisome features" (e.g., a cyst diameter $\geq 3 \mathrm{~cm}$, thickened cyst walls, or an MPD diameter of 5-9 mm) should undergo EUS-FNA; if no high-risk feature or positive cytology (malignancy/high-grade dysplasia) is documented, surveillance is indicated according to cyst size [10]. 

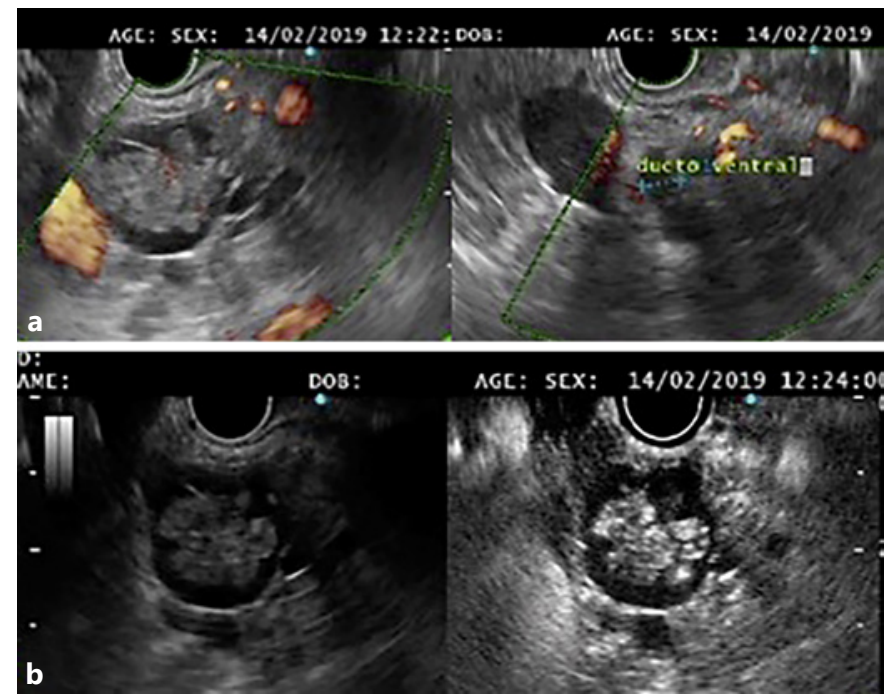

Fig. 1. a A branch-duct intraductal papillary mucinous neoplasm communicating with the ventral main pancreatic duct, with a large solid nodule. b Contrast enhancement of the nodule was documented with intravenous administration of SonoVue ${ }^{\circledR}$.

All the consensus guidelines on the management of pancreatic cystic neoplasms state that EUS-guided cyst ablation should not currently be performed without a dedicated investigation protocol [5, 10-12]. Promising data regarding the safety and efficacy of alcohol-free chemoablation and radiofrequency ablation (RFA), at least for a subset of mucinous cysts, has recently emerged, but technical refinement and validation are still required [6-8].

Selection Criteria for the Ablation of Pancreatic Cystic Neoplasms

Indications. Pancreatic cyst ablation, either by injection of an ablative agent or radiofrequency, can be considered for potentially pre-malignant cysts, particularly mucinous cysts with worrisome features in patients who are not candidates for surgery, or as an alternative for patients who refuse surgery but have a reasonable life-expectancy [8]. EUS-FNA (for cytology and cyst fluid analysis, including carcinoembryonic antigen [CEA]) should be used as an adjunct to MRI for the characterization of cystic lesions before ablation therapy $[6,8]$. Some characteristics of the targeted cyst may favour the choice of one ablation modality over the other. Criteria for ablation are better defined for EUS-guided injection therapy [8] and include:
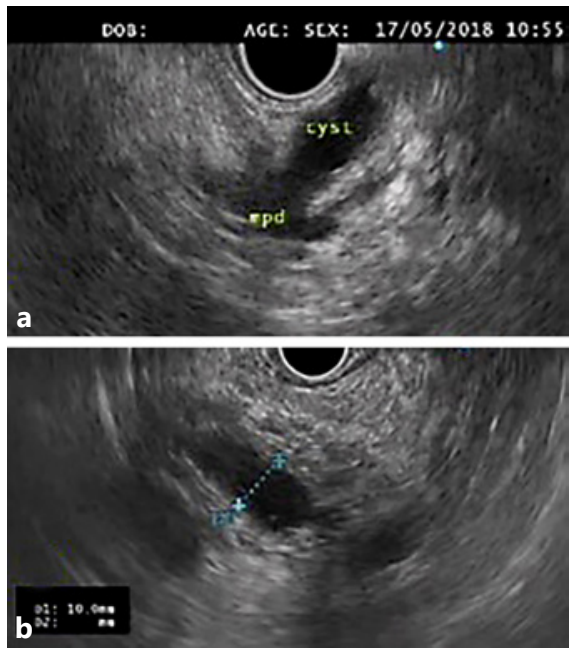

Fig. 2. a A mixed-type intraductal papillary mucinous neoplasm of the pancreatic body. b An elongated cyst communicating with a significantly dilated $(10 \mathrm{~mm})$ main pancreatic duct was visible on endoscopic ultrasound.

- Unilocular or oligolocular cysts ( $<6$ locules) with a presumed or confirmed diagnosis of a mucinous pancreatic cyst (mucinous cystadenoma or BD-IPMN).

- Pancreatic cysts with a diameter $>3 \mathrm{~cm}$ or enlarging pancreatic cysts with a diameter $>2 \mathrm{~cm}[6,8]$.

The best results for EUS-guided injection for cyst ablation are achieved with unilocular cysts not communicating with the MPD and measuring $2-6 \mathrm{~cm}$ in size, with the best response rate at the smaller end of the range [13]. In fact, the ideal candidate for injection therapy is a unilocular mucinous cystadenoma measuring 20-60 mm [13]. Poor results are obtained in cysts $>60 \mathrm{~mm}$ and with several locules [8]. For RFA, the criteria also include mucinous cysts (BD-IPMNs or mucinous cystadenomas) measuring up to $6 \mathrm{~cm}$ in size [7]. The ultimate goal of ablation is to completely destroy the neoplastic lining of the cyst, to eliminate it and ultimately decrease the likelihood of progression to invasive cancer [8]. The presence of $>6$ locules and a mural nodule are predictors of incomplete cyst ablation with chemoablation [8]. In these cases, RFA will possibly achieve a better ablative effect [7]. In a recent prospective trial of 17 cysts (mostly BD-IPMNs with mural nodules) submitted to RFA, results were encouraging, with complete resolution of all 12 mural nodules 1 year after ablation [7].

Contraindications. A dilated MPD $>5 \mathrm{~mm}$ and a clear open communication of the cyst with the MPD are rela- 
tive contraindications for chemoablation, because of the increased risk of injectate leakage to the MPD and injury of the duct $[6,8]$. Nevertheless, BD-IPMNs can be selected for ablation, providing the cyst is not directly adjacent to the MPD and there is no visible communication with the MPD during aspiration (the volume in the syringe is larger than the anticipated volume) and injection (i.e., fluid exiting the cyst) [8].

As previously stated, a significant solid component (a mural nodule) within the cyst is also a relative contraindication for injection therapy, because of the poor results [8]. In the presence of a mural nodule, RFA may be a better option, achieving in most cases complete resolution of the mural nodule 1 year after ablation [7].

General absolute contraindications for ablation are similar to other endoscopic procedures, including coagulopathy. Specific contraindications to the procedure include overt signs of malignancy, previous acute pancreatitis (which increases the risk of MPD injury) and a short life-expectancy (since the anticipated benefit from the procedure is limited) [8].

\section{Technical Aspects}

For chemo-ablation, a $19-\mathrm{G}$ or $22-\mathrm{G}$ standard FNA needle can be used for aspiration and injection, but if the cyst is easily accessible (as in the pancreatic body or tail), a $19-\mathrm{G}$ needle is preferable $[6,8]$. A larger-diameter needle allows for easier aspiration of the mucinous fluid and easier injection of the chemotherapy drugs (since paclitaxel is quite viscous) $[6,8]$. Before ablation, the cyst fluid should be aspirated almost completely. The amount of cyst fluid aspirated should be recorded and the same amount of ablative agent infused $[6,8,13]$.

Ethanol is the traditionally used agent for ablation and was evaluated in several randomized controlled trials (RCTs) during the last decade $[8,13,14]$. However, it should probably not be used anymore as an ablative agent, due to its low efficacy and high rate of adverse events, with acute pancreatitis reported in up to $10 \%$ of cases $[8,13$, 14].

In the most recent trials, the ablation solution includes paclitaxel at a $3 \mathrm{mg} / \mathrm{mL}$ concentration, which is injected and left inside the cyst $[8,13,15]$. Two recent RCTs showed that ethanol may increase the rate of complications and is not required for effective cyst ablation when a multiagent ablation solution of paclitaxel $(3 \mathrm{mg} / \mathrm{mL})+$ gemcitabine $(19 \mathrm{mg} / \mathrm{mL})$ is used $[6,15]$.

To prevent post-procedural infection, prophylactic antibiotics (fluoroquinolone or $\beta$-lactam) are recommended during ablation and should be continued for $3-5$ days $[7,8]$.
There are 2 radiofrequency devices available for current practice: a dedicated needle-RFA device (STARmed; Taewoong, Seoul, Korea) and the Habib probe, a 1-Fr probe introduced via a $19-\mathrm{G}$ needle (Habib EndoHBP, EMcision). The STARmed device was used in the most relevant trials analysing RFA [7]. If the STARmed device is used, after targeting the cyst with the RFA needle, $50 \mathrm{~W}$ is applied with the continuous-mode setting until reaching 100- $\Omega$ impedance or when white bubbles appear alongside the needle and outside the targeted lesion. Where possible, a 2-mm distance should be kept between any critical surrounding structures (like the common bile duct and the Wirsung duct) and the tip of the active part [7]. Prior to RFA, aspiration of most of the intra-cystic fluid (using a 22-G or 19-G needle, until a thin layer of fluid remains) is recommended to decrease damage to the adjacent parenchyma (due to diffusion of the heat through the fluid) [7].

In RFA of cystic lesions, some groups also recommend prophylaxis of acute pancreatitis with rectal diclofenac [7].

\section{Efficacy and Safety Data}

In published series, both alcohol-free chemoablation and RFA have a low rate of adverse events $[6-8,13,15]$. In chemoablation, most of the reported adverse events are related to the use of ethanol $[6,8,13-16]$. In the 2 recent RCTs that showed that ethanol is not required for effective cyst ablation, using a multi-agent ablation solution of paclitaxel $(3 \mathrm{mg} / \mathrm{mL})+$ gemcitabine $(19 \mathrm{mg} / \mathrm{mL})$, the overall rate of complete ablation at 12 months was $64 \%$ (similar to the ethanol control group) $[6,15]$. Importantly, serious adverse events within 30 days after the procedure occurred in $6 \%$ of patients in the control group versus none in the alcohol-free group [6]. Minor adverse events occurred in $22 \%$ of patients in the control group and none in the alcohol-free group $(p=0.01)$ [6]. In summary, the best protocol for injection therapy of pancreatic cysts (preferably, alcohol-free) is not totally defined and different study groups have tested variable doses and volumes of paclitaxel [16]. The most encouraging results have been achieved with multi-agent chemoablation, including paclitaxel (at a $3 \mathrm{mg} / \mathrm{mL}$ concentration) and gemcitabine (at a $19 \mathrm{mg} / \mathrm{mL}$ concentration) $[6,15]$. The value of repeat treatment has not yet been studied [16].

Regarding RFA of pancreatic cysts, in a recent prospective multi-centre study involving 17 cysts (16 IPMNs and 1 mucinous cystadenoma; mean size $28 \mathrm{~mm}$, range 9-60 mm), 65\% had disappeared completely at 12 months and all 12 mural nodules showed complete resolution [7]. 
There are currently no randomized studies comparing pancreatic cyst ablation versus no treatment or surgery. Most importantly, the clinical impact, i.e., a reduction in the incidence of pancreatic adenocarcinoma, has not yet been shown [16]. Hence, there is no clear evidence that the postulated survival benefits outweigh the risks associated with the procedure and institutions performing the procedure should do so under a research protocol [8].

The goal of EUS-guided pancreatic cyst ablation is to completely destroy the neoplastic lining of the cystic tumour, which can only be assessed following surgical resection of the cyst. Radiologic cyst resolution (on CT/ MRI) is a surrogate outcome of EUS-guided ablation, which is achieved in about $70 \%$ of cases with chemoablation [6]. However, the complete epithelial ablation rate is still unknown $[6,8]$.

After pancreatic cyst ablation, patients should be followed up to assess for recurrence and/or worrisome morphological changes, preferably by MRI at 6-month intervals for the first year, and then annually until no longer warranted due to a patient's age and medical condition. In the case of cyst recurrence, surgery or retreatment may be considered $[8,13]$.

Some major concerns regarding EUS-guided ablation of pancreatic cysts, which prevent its current use in clinical practice outside a research protocol, must be stated:

1 Chemoablation seems less effective in cysts with highrisk/worrisome features: lower rates of radiologic response were documented in larger cysts $(>35 \mathrm{~mm})$ and in cysts with a mural nodule [13]. Also, involvement of the MPD (a dilated MPD >5 mm and a clear open communication of the cyst with the MPD) is a contraindication for chemoablation (because of the increased risk of injectate leakage to the MPD and injury of the duct) $[6,8,15]$.

2 The protocol for ablation (preferably, alcohol-free) is not defined $[6,8,15]$.

3 Radiologic cyst resolution is a surrogate outcome of EUS-guided ablation (achieved in $60-70 \%$ of cases with chemoablation), but the complete epithelial ablation rate is unknown $[6,15,16]$.

4 The clinical impact, i.e., progression to malignancy of pancreatic cysts is avoided, has not yet been shown [16].

5 The best candidates for ablation are still to be defined. Pancreatic cyst ablation can be considered for patients who are not candidates for surgery, but the clinical benefit of ablating a cyst that usually follows an indolent course in a patient unfit for surgery is questionable.

\section{Key Points}

- EUS-guided cyst ablation should not currently be performed without a dedicated investigation protocol.

- Both alcohol-free chemoablation and RFA seem to be safe and promising minimally invasive options for a subset of pre-malignant pancreatic cysts, but still require technical refinement and validation.

- Pancreatic cyst ablation can be considered for patients with BD-IPMNs or mucinous cystadenomas $(2-6 \mathrm{~cm})$ who are not candidates for surgery but have a reasonable life-expectancy, or as an alternative for patients who refuse surgery.

- Ethanol should probably not be used as an ablation agent, due to its low efficacy and a high rate of adverse events.

- The best protocol for chemoablation (preferably, alcohol-free) still needs to be defined.

- A dilated MPD $>5 \mathrm{~mm}$ and a clear open communication of the cyst with the MPD are relative contraindications for chemoablation, because of the increased risk of injectate leakage to the MPD and injury of the duct.

- A significant solid component (mural nodule) within a pancreatic cyst is a relative contraindication for chemoablation, due to poor results. In this setting, RFA may be a better choice.

- Prophylactic antibiotics are recommended during ablation and should be continued for 3-5 days.

- Long-term clinical efficacy of EUS-guided ablation in different types of cysts is still not defined.

\section{Ablation of pNETs}

Incidental diagnosis of small pNETs $(\leq 2 \mathrm{~cm})$ has greatly increased in the last years [17]. These tumours are mostly non-functional and indolent, although a significant proportion (up to 39\%) may reveal an aggressive behaviour despite their small size [18]. Therefore, there is still no consensus on the best management for non-functional tumours $<2 \mathrm{~cm}$ and institutional experiences may be quite variable [19]. The risks of under-/overtreatment should be carefully evaluated with the patient and balanced with the potential morbidities related to surgery [18]. Current guidelines consider only 2 opposite approaches for these small lesions, jumping from observation to surgery, and advocating surgery even for some symptomatic pNETs with a low malignant potential, such as insulinomas $[18,19]$. In fact, management decisions about small pNETs can be challenging and must consider 
the clinical presentation as well as size, grade, and location of the tumour within the pancreas, together with the patient's health status, age, and wishes [18].

EUS-FNB is helpful to confirm the diagnosis of a pNET and to ascertain tumour grade, which is particularly relevant in non-functional pNETs $1-2 \mathrm{~cm}$ in size $[18,19]$. EUS-guided treatments for pNETs are still investigational, with promising results obtained with RFA, particularly in the treatment of insulinomas, but also in non-functional pNETs $\leq 2 \mathrm{~cm}[7,20]$.

\section{Selection Criteria for Ablation of pNETs}

Only patients with pNETs $\leq 2 \mathrm{~cm}$, unfit for or refusing surgery, should be considered for EUS-guided ablation $[7,20]$.

Functional pNETs represent a minority (10\%) of all pNETs and the risk of malignancy varies depending on the type; it is low (5-15\%) for insulinoma but high for other types [19]. Insulinomas are usually small $(\leq 2 \mathrm{~cm})$, solitary, benign lesions, equally distributed between the head, body, and tail of the pancreas, and have probably the best indication for EUS-guided ablation as an alternative to surgery [20]. Functional pNETs secreting glucagon, vasoactive intestinal peptide (VIP), and adrenocorticotropic hormone (ACTH) are particularly aggressive [20] and EUS-guided ablation is a less suitable option for these tumours.

\section{Technical Aspects}

As stated above, the STARmed device was used in the most relevant trials analysing RFA of pNETs [7]. This technique is similar to that previously described for the ablation of pancreatic cysts $[7,20]$.

EUS-guided RFA seems to be safe, with a rate of adverse events of $3.5 \%$ when important preventive measures are taken, namely: (1) prophylactic antibiotics, (2) prophylaxis of acute pancreatitis with rectal diclofenac, and (3) aspiration of most of the intra-cystic fluid by FNA prior to ablation in cystic lesions [7].

EUS-guided injection of ethanol carries the risk of uncontrolled diffusion [21]. EUS-guided RFA ensures a more controlled ablative effect, inducing tumour necrosis and enhancing the anti-tumoral immune response [7].

\section{Efficacy and Safety Data}

In the largest prospective series, which included 14 pNETs $(10-20 \mathrm{~mm})$, the rate of complete resolution after 1 year was $86 \%$ [7]. Importantly, there can be a delayed response after RFA of a pNET (even after 6 months), possibly due to stimulation of the immune response by the release of antigens by the necrotic tissue $[7,22]$. In the treatment of insulinomas, EUS-guided RFA showed a quick symptomatic improvement and sustained results at 2 years of follow-up in a small series of 3 patients [20]. Long-term results of EUS-guided treatment of pNETs are still not defined and the surveillance protocol should not be changed even after complete imagiological resolution of the lesion [7].

\section{Key Points}

- EUS-guided treatment of pNETs is still investigational, with promising results obtained with RFA, particularly for insulinomas, but also for nonfunctional pNETs $\leq 2 \mathrm{~cm}$.

- Only patients with pNETs $\leq 2 \mathrm{~cm}$ who are unfit for or refuse surgery should be considered for EUS-guided ablation.

- Functional pNETs secreting glucagon, VIP, and ACTH are particularly aggressive and EUS-guided ablation should probably not be performed on these tumours.

- There can be a delayed response after RFA of a pNET (even after 6 months), possibly due to stimulation of the immune response by the release of antigens by the necrotic tissue.

- The long-term results of EUS-guided treatment of pNETs have still not been defined and the surveillance protocol should not be changed even after complete imagiological resolution of the lesion.

\section{Statement of Ethics}

The final manuscript was revised and approved by all the members of the Governing Board of GRUPUGE.

\section{Disclosure Statement}

All authors disclose there are no personal conflicts of interest or financial relationships relevant to this publication.

\section{Funding Sources}

There was no funding.

\section{Author Contributions}

M.B.: article concept and design, literature review, and draft of the manuscript. A.C., S.L., S.M., T.M., P.M.-R., and N.N.: literature review and critical review of the manuscript. 


\section{References}

1 Farrell JJ. Prevalence, Diagnosis and Management of Pancreatic Cystic Neoplasms: Current Status and Future Directions. Gut Liver. 2015 Sep;9(5):571-89.

2 Lee DW, Kim MK, Kim HG. Diagnosis of pancreatic neuroendocrine tumors. Clin Endosc. 2017 Nov;50(6):537-45.

3 Wu BU, Sampath K, Berberian CE, Kwok KK, Lim BS, Kao KT, et al. Prediction of malignancy in cystic neoplasms of the pancreas: a population-based cohort study. Am J Gastroenterol. 2014 Jan;109(1):121-9.

4 Sachs T, Pratt WB, Callery MP, Vollmer CM Jr. The incidental asymptomatic pancreatic lesion: nuisance or threat? J Gastrointest Surg. 2009 Mar;13(3):405-15.

5 Elta GH, Enestvedt BK, Sauer BG, Lennon AM. ACG Clinical Guideline: Diagnosis and Management of Pancreatic Cysts. Am J Gastroenterol. 2018 Apr;113(4):464-79.

6 Moyer MT, Sharzehi S, Mathew A, Levenick JM, Headlee BD, Blandford JT, et al. The Safety and Efficacy of an Alcohol-Free Pancreatic Cyst Ablation Protocol. Gastroenterology. 2017 Nov;153(5):1295-303.

7 Barthet M, Giovannini M, Lesavre N, Boustiere C, Napoleon B, Koch S, et al. Endoscopic ultrasound-guided radiofrequency ablation for pancreatic neuroendocrine tumors and pancreatic cystic neoplasms: a prospective multicenter study. Endoscopy. 2019 Sep; 51(9):836-42.

8 Teoh AY, Seo DW, Brugge W, Dewitt J, Kongkam P, Linghu E, et al. Position statement on EUS-guided ablation of pancreatic cystic neoplasms from an international expert panel. Endosc Int Open.2019 Sep;7(9):E106477.

9 Moutinho-Ribeiro P, Liberal R, Macedo G. Endoscopic ultrasound in pancreatic cancer treatment: facts and hopes. Clin Res Hepatol Gastroenterol. 2019 Oct;43(5):513-21.

10 Tanaka M, Fernández-Del Castillo C, Kamisawa T, Jang JY, Levy P, Ohtsuka T, et al. Revisions of international consensus Fukuoka guidelines for the management of IPMN of the pancreas. Pancreatology. 2017 Sep - Oct; 17(5):738-53

11 European Study Group on Cystic Tumours of the Pancreas. European evidence-based guidelines on pancreatic cystic neoplasms. Gut. 2018 May;67(5):789-804.

12 Muthusamy VR, Chandrasekhara V, Acosta $\mathrm{RD}$, Bruining DH, Chathadi KV, Eloubeidi MA, et al.; ASGE Standards of Practice Committee. The role of endoscopy in the diagnosis and treatment of cystic pancreatic neoplasms. Gastrointest Endosc. 2016 Jul;84(1):1-9.

13 Choi JH, Seo DW, Song TJ, Park DH, Lee SS, Lee SK, et al. Long-term outcomes after endoscopic ultrasound-guided ablation of pancreatic cysts. Endoscopy. 2017 Sep;49(9): $866-73$.

14 Gómez V, Takahashi N, Levy MJ, McGee KP, Jones A, Huang Y, et al. EUS-guided ethanol lavage does not reliably ablate pancreatic cystic neoplasms (with video). Gastrointest Endosc. 2016 May;83(5):914-20.

15 Moyer MT, Dye CE, Sharzehi S, Ancrile B, Mathew A, McGarrity TJ, et al. Is alcohol required for effective pancreatic cyst ablation? The prospective randomized CHARM trial pilot study. Endosc Int Open. 2016 May; 4(5):E603-7.

16 Yachimski P, Varadarajulu S. Endoscopic Ultrasound-Guided Pancreatic Cyst Ablation: More Peril Than Promise? Gastroenterology. 2017 Nov;153(5):1183-5.

17 Dasari A, Shen C, Halperin D, Zhao B, Zhou $\mathrm{S}, \mathrm{Xu} \mathrm{Y}$, et al. Trends in the incidence, prevalence, and survival outcomes in patients with neuroendocrine tumors in the United States. JAMA Oncol. 2017 Oct;3(10):1335-42.

18 Bartolini I, Bencini L, Risaliti M, Ringressi MN, Moraldi L, Taddei A. Current Management of Pancreatic Neuroendocrine Tumors: From Demolitive Surgery to Observation. Gastroenterol Res Pract. 2018 Jul;2018: 9647247.

19 Howe JR, Merchant NB, Conrad C, Keutgen XM, Hallet J, Drebin JA, et al. The North American Neuroendocrine Tumor Society Consensus Paper on the Surgical Management of Pancreatic Neuroendocrine Tumors. Pancreas. 2020 Jan;49(1):1-33.

20 Lakhtakia S, Ramchandani M, Galasso D, Gupta R, Venugopal S, Kalpala R, et al. EUSguided radiofrequency ablation for management of pancreatic insulinoma by using a novel needle electrode (with videos). Gastrointest Endosc. 2016 Jan;83(1):234-9.

21 Lakhtakia S, Seo DW. Endoscopic ultrasonography-guided tumor ablation. Dig Endosc. 2017 May;29(4):486-94.

22 Slovak R, Ludwig JM, Gettinger SN, Herbst RS, Kim HS. Immuno-thermal ablations boosting the anticancer immune response. J Immunother Cancer. 2017 Oct;5(1):78. 